\title{
Evaluation of the suitability of IR and TV image aggregation algorithms for the purposes of welding process assessment ${ }^{*}$
}

by W. Jamrozik and M. Fidali

Dept. of Fundamentals of Machinery Design, Silesian University of Technology, Konarskiego 18a, 44-100 Gliwice, Poland, \{wojciech.jamrozik; marek.fidali\}@polsl.pl

\begin{abstract}
This paper focuses on the evaluation of IR and TV image fusion methods. Input images were acquired during the MIG/MAG welding process and represent an area of the welding arc as well as the heat-affected zone.

In order to choose the fusion method, which produced the most suitable images for further welding process diagnostics a different fusion methods were tested and evaluated. Subjective and objective methods (with and without reference image) were taken into consideration. The obtained results show that there are some inconsistencies when different evaluation methods are applied, however the most promising fusion method can be chosen for this particular case.
\end{abstract}

\section{Introduction}

Machine vision as a promising tool for industrial processes monitoring and control, is incorporated more and more often in many different areas of industry. Until recently machine vision systems have mainly utilized one or more cameras that take images in the visible light range. Development of industrial infrared cameras market has contributed to the increase of use of those cameras in machine vision systems.

Imaging devices, that made acquisition in the range of infrared radiation are often equipped in integrated visible light cameras or work simultaneously with such cameras. At least two reasons of simultaneous infrared and visible light acquisition of the same scene can be indicated. The first of them arise from the need of aiding the thermograms interpretation by human operator. The latter one is connected with the possibility of effectiveness evaluation of phenomena that occurred in the observed scene area through taking simultaneously into account information carried by the infrared and visible light radiation, recorded in the form of digital images.

As the example of industrial process, which can be assessed on the basis of information gathered in various electromagnetic radiation bands welding process can be given. During the welding process, welding arc, heated to the high temperature welding gasses and metal fumes, welded elements, electrode material, etc. generate high amount of ultraviolet, visible light and infrared radiation, which can be used in different ways for process diagnostic [30]. In order to assess the welding process, among others solutions, vision systems that are working in the infrared and visible light range could be utilized. Such systems allow monitoring and assessment of the welding process stability [1][13][21], evaluation of size and placement of the welding pool [15][18][25], as well as control of the joint geometry and the quality of welded seam [3][9][1][7][10][11].

The results presented in various publications, besides high effectiveness of the proposed solutions, show also, that information contained in thermograms (e.g. about the temperature distribution in the heat affected zone) can usefully complement information contained in visible light image. This is the premise to joint analysis of infrared and visible light images, simultaneously taken during the welding process. One of the methods used to combine information present in visible and infrared light images is the image fusion.

Image fusion is a technique which leads to the formation of one synthetic image on the basis of at least two input images originated from different sources. There are many various method of image fusion. Those methods are mainly based on the following three basic operations: image registration, image aggregation and in some cases deblurring. Simple fusion method is built in some models of modern infrared cameras, as well as in software dedicated to infrared image analysis. The methods consist in image aggregation through weighted averaging of input image pixel values. The weighting coefficient values can be modified interactively. That allows the fluent control of the information content that is put in the output, fused image. Such simple method is suitable for images of static scene and when acquisition and image manipulation parameters are fully controlled by operator. Previous research of the authors [14] showed that in the case of image fusion techniques application for automatic diagnostic of dynamic processes, e.g. welding process, it is necessary to choose most suitable registration and aggregation method. Using right methods result in the obtainment of fused images that contain maximal amount of relevant information which is demanded for observed process assessment.

In the paper results of research connected with the search for group of most suitable image aggregation methods, used to combine visible light and infrared images taken during the MIG/MAG welding process were presented. In order to assess the quality of each of the selected methods, objective measures as well as subjective welding expert

\footnotetext{
* Scientific work partially financed from resources assigned to science in the years 2009-2012 as a research project N504281937 and from resources assigned to statutory activity of Dept. of Fundamentals of Machinery Design, Silesian University of Technology at Gliwice.
} 
estimates were considered. Image fusion was performed for sequences of images taken during several experiments with simulation of different process states caused by intentionally introduced instabilities of process parameters.

\section{Image fusion}

Computer image fusion is an algorithmic technique of synergistic combination of information coded in two or more digital images. The result of image fusion is a new, synthetic image, characterized by relevant features of input images and a limited number of redundant features. Computer image fusion can be used in various fields of science and technology [17][26] which resulted in the formation of wide spectrum of image combination algorithms. Those algorithms can be divided in three groups: pixel level algorithms, feature level algorithms and symbolic level algorithms.

In practical applications most often algorithms working on the lowest level of abstract, namely the pixel level are used. This follows from the way they perform the combination. It assumes the existence of the relation between corresponding pixels of input images. As a consequence, results given by pixel level algorithm are strongly bounded with the quality of geometrical alignment determined in the registration process.

Image registration is, besides the aggregation, one of key stages in most of image fusion algorithms. Many image registration methods were elaborated in the last decades. Depending upon the chosen feature space, image registration methods can be divided into two groups: area-based and feature-based (landmark-based) ones. Area-based methods use image regions with similar intensity for registration, whereas feature-based ones use salient features like point, edges, etc. Each registration method demands the selection of transformation model between images, which best describes the degradations between content in input images. In this case translational transformation, affine transformation, elastic transformation or free transformation can be used.

In the case of visible light and infrared images being the source of multimodal information, process of registration is not a trivial task. The main reason of major difficulties is the definition of geometrical relation between object being observed and represented on each input image. This is caused by differences in radiation distribution in infrared and visible light band. There can be no correspondence between the intensity (gray level, colour) of the objects represented in the TV image and the infrared one which is visualised on the thermogram with use of the colour map assigning the luminance (grey level or colour) to all thermopixels. Difficulties in registration could be compounded by incorrect choice of colour map and temperature scale. Additionally, registration methods must be robust in terms of common degradations caused by the offset, pitch and roll between optical axes of cameras used in particular system and differences in resolution of acquired images.

Review of numerous publications [8][12][32] and authors' study [14] on registration methods lead to the conclusion, that in the case of visible light and infrared images it is advisable to use hybrid methods based on area information and on the image features as well. One of suitable methods for registration of greyscale or indexed images is using the entropy based objective function. It also takes into consideration the 3D histogram build for the edge orientation maps and pixel intensities values [16]. Objective function represents the uncertainty about the edge orientation coincidence of the corresponding intensity pair as well as the uncertainty between the intensity values of two images. In [30] the authors carried the assessment of several image registration algorithms for visible light images and thermograms fusion purposes. The obtained results proved that the above presented edge orientation map algorithm gives best results. Therefore, this algorithm was used for image registration of images used in the presented research.

After image registration, the next step, being the main object of the research, is the aggregation of information contained in input images. Aggregation process is an operation of joining several previously registered images in one output image. There are various classes of aggregation methods, but for the purpose of the presented research, it was assumed that only pixel-based multiscale algorithms were taken into consideration.

Multiscale transformation is one of steps realized within image aggregation method. It divides the image in a hierarchical structure where each level is a transformed version of image on the previous level. Images in the structure are ordered from original one (the highest resolution) to the transformed one with smallest resolution. Hierarchical structures are obtained by application of one of two basic transforms:

- $\quad$ pyramidal transform, which generates a hierarchical structure with a pyramid shape, in the way that image on the certain pyramid stage has a twice greater size than the image on the higher stage,

- $\quad$ wavelet transform, similar to the pyramidal approach, but the result is a relevant (not redundant) image representation.

Operation of hierarchical structure generation is called decomposition. It is the first of three aggregation stages. Next stages that lead to the obtainment of aggregated image are as follows:

- joining of images on corresponded stages of the hierarchical structure which was obtained in the decomposition stage. This operation is described by the aggregation rule applied for all input images on each decomposition stage. Aggregation rules can be applied to certain pixels, areas or regions. In each case the rule is based on some selection or averaging operator;

- $\quad$ synthesis, the inverse transformation to one used on the decomposition stage. The synthesis result is the output image obtained from the decomposed but aggregated hierarchical structure.

For research purposes in image fusion methods following aggregation algorithms were used:

1. Method based on the average operator (MEA) - in that method pixel-wise operator is used to average corresponding pixel values from all input images. This is a reference method without use of pyramidal transformation. 
2. Method based on principal component analysis (PCA) [20] - in that method a pca analysis is used at each pyramid level with decimated images.

3. Nonlinear maximum operator (MAX) - second non hierarchical method, where output pixel is select as the pixel with maximal luminance values from corresponding set of corresponding pixels in input images.

4. Nonlinear minimum operator (MIN) - third non hierarchical method, where output pixel is select as the pixel with minimal luminance values from corresponding set of corresponding pixels in input images.

5. Laplace pyramid method (LAP) [5] - in this method where images on following pyramid level are band filtered and decimated copies of images on the pyramid lower level.

6. Filter-subtract-decimate (FSD) [2] - is more computational efficient modification of the Laplace pyramid method.

7. Ratio pyramid method (RAT) [29] - images on the subsequent decomposition pyramid stages are ratio of two following stages of Gaussian pyramid.

8. Contrast pyramid method (CON) [28] - method similar to RAT method. The main difference is in subtraction of unitary background image form image on lower pyramid level before the ratio calculation.

9. Gradient pyramid method (GRA) [6] - images on Laplace pyramid levels are completely represented by a set of four gradient pyramids, derivates in horizontal, vertical and two diagonal directions.

10. Method based on discrete wavelet decomposition (DWB) [17] - in this method where image decomposition is performed by discrete wavelet transform through recursive low- and highpass filtration in two directions with DBSS $(2,2)$ wavelet.

11. Method based on shift invariant wavelet transform (SIH) [23] - input images are decomposed into a shift invariant wavelet structures using the wavelet frames concept with the Haar wavelet

12. Pyramid of morphological differences (MOR) [22] - each pyramid level is the difference between image at the same level of Gaussian pyramid and the same image after morphological operations such as opening, closing and dilate.

In all the considered hierarchical methods hybrid aggregation rule which differentiates between selection and averaging is incorporated

\subsection{Obtaining images for fusion purposes}

For the image fusion purposes visible light and infrared images of welding arc were used. Images were taken during the series of experiments performed on a laboratory stand (Fig. 1) consisting of:

- $\quad$ Mechanised welding stand for rectilinear MIG/MAG welding

- Microprocessor controlled machine for pulsed and standard MIG/MAG welding Castolin TotalArc 5000

- System for monitoring and acquisition of welding process parameters: current, voltage, welding speed and shield gas flow,

- Vision system consists of infrared camera (Infratec VarioCam Head, 640x480px, lens $\mathrm{f}=50 \mathrm{~mm}$ ) and visible light camera (ImagingSource DMK21AF04, 640x480px, lens $\mathrm{f}=25 \mathrm{~mm}$ ) observing the welding arc and infrared camera (Infratec VarioCam $320 \times 240 p x$, lens $f=50 \mathrm{~mm}$ ) for welded joint observation. There were also computers with proper software which allows image acquisition with speed $50 \mathrm{fps}$. Both the images and the process parameters were acquired synchronically.

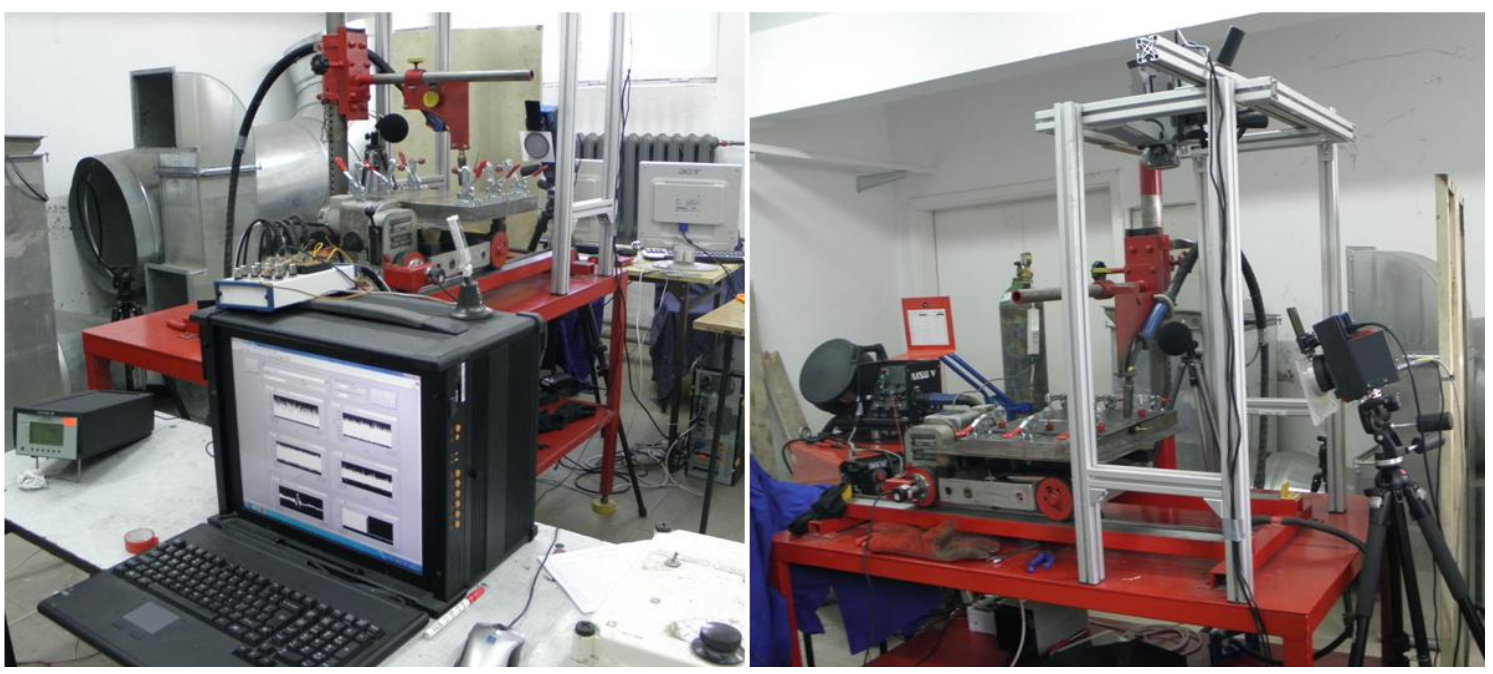

Fig. 1. Laboratory stand 
During the experiment a groove weld was made between plates made of steel S235JR (EN 10027-1) with dimensions $300 \times 150 \times 5 \mathrm{~mm}$. Apart from special cases, the edges of most of the joined plates bevelled at an angle of $\alpha=60^{\circ}$ and the offset between them was $b=1,0 \mathrm{~mm}$.

Groove welds were made on the copper base equipped with a mechanical clamping system for joined plates. For welding a solid electrode wire with a diameter of 0,2 mm (Castolin CastoMag 45255) and a shield gas M21 $\left(82 \% \mathrm{Ar}+18 \% \mathrm{CO}_{2}\right)$ were used. Nominal welding parameters are presented in Tab. 1.

Table 1. Optimal MIG/MAG welding parameters

\begin{tabular}{|c|c|c|c|c|c|}
\hline $\begin{array}{c}\text { Welding current } \\
{[\mathrm{A}]}\end{array}$ & $\begin{array}{c}\text { Welding voltage } \\
{[\mathrm{V}]}\end{array}$ & $\begin{array}{c}\text { Welding speed } \\
{[\mathrm{cm} / \mathrm{min}]}\end{array}$ & $\begin{array}{c}\text { Wire feeding rate } \\
{[\mathrm{m} / \mathrm{min}]}\end{array}$ & $\begin{array}{c}\text { Shield gas flow } \\
{[\mathrm{l} / \mathrm{min}]}\end{array}$ & $\begin{array}{c}\text { Electrode outlet } \\
{[\mathrm{mm}]}\end{array}$ \\
\hline 240 & 25 & 32 & 7,4 & 15 & 15 \\
\hline
\end{tabular}

In the experiment several welding process faults were simulated and thus the influence on the welded joint quality was made. On the basis of performed simulations there were 12 different states of the welding process, defined as follows:

S1 - State of welding process obtained with nominal welding parameters, allowing obtainment of welding joint which can be classified into one of three quality levels (B, C, D) according to the standard EN-ISO 5817

S2 - State of welding process obtained through the decay of the shielding gas flow, resulting in the formation of welded joint not meeting any quality level according to the ISO 5817 ,

S3 - State of welding process obtained during welding of plates with distinct outbreaks of atmospheric corrosion on the welded surfaces. Welded joint obtained during this process does not meet any quality level according to ISO 5817 ,

S4 - State of the welding process developed during welding process with wire electrode whose surface was covered by oxide layer caused by atmospheric corrosion, resulting in the formation of welded joint that does not meet any quality level according to ISO 5817,

S5 - State of the welding process caused by irregularities of the edge of the joined plates from side of the weld root, resulting in the formation of welded joint that does not meet any quality level according to ISO 5817 ,

S6 - State of the welding process caused by the existence of oil contamination on joined metal surfaces and, resulting in the formation of welded joint that does not meet any quality level according to ISO 5817,

S7 - State of the welding process caused when the welding current deviation from the nominal value about $\pm 20 \%$ and resulting in the formation of welded joint that does not meet any quality level according to ISO 5817 ,

S8 - State of the welding process obtained during the welding of plates with different offset intervals of $1,5 \mathrm{~mm}, 2,0 \mathrm{~mm}$, $2,5 \mathrm{~mm}, 3,0 \mathrm{~mm}$ and $3,5 \mathrm{~mm}$ resulting in the formation of welded joint that does not meet any quality level according to ISO 5817,

S9 - State of the welding process caused by the welding voltage deviation from the nominal value about $\pm 15 \%$ and resulting in the formation of welded joint that does not meet any quality level according to ISO 5817 ,

S10 - State the welding process, obtained during welding of steel sheets with improper welding groove geometry resulting from a change in the angle of bevel on $0^{\circ}, 15^{\circ}, 30^{\circ}, 45^{\circ}, 60^{\circ}$ and resulting in the formation of welded joint that does not meet any quality level according to ISO 5817,

S11 - State of the welding process caused by welding speed deviation from the nominal value about $-20 \%$ and $+50 \%$ and resulting in the formation of welded joint that does not meet any quality level according to ISO 5817 ,

S12 - State of the welding process obtained during automatic welding when the wire feeder is equipped with worn rollers and resulting in the creation of connections that do not meet any quality level according to ISO 5817.

A total of 91 welds were made. For each of the specified conditions at least seven experiments were performed. During each experiment sequences of infrared and visible light images were acquires. For image fusion purposes each image in the sequences was pre-processed by application of the following operation: the selection of a region of interest and resizing.

Figure 2 presents an example of images of the arc taken in the visible light and infrared band. Figure 3 shows the effect of the fusion of images shown in Figure 2 with the use of different aggregation methods of the information contained in the input images. 

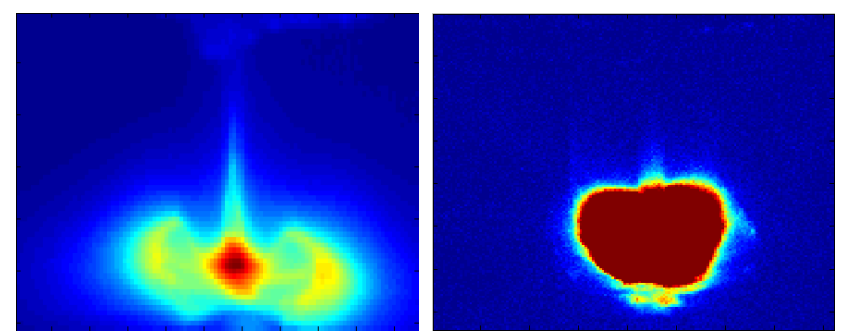

Fig. 2. Infrared and visible light input images for fusion process

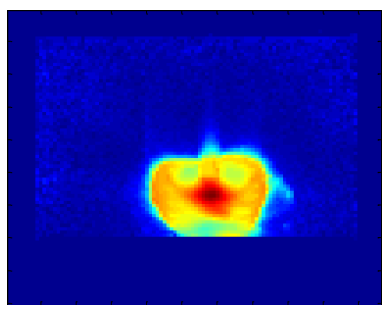

a)

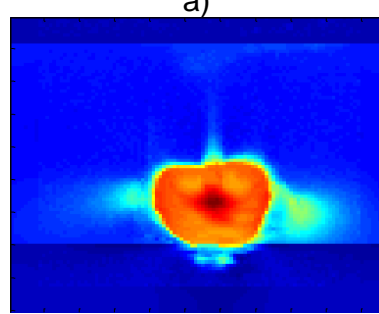

e)

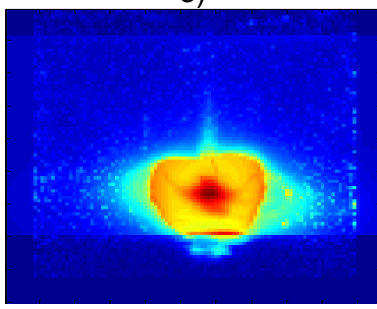

i)

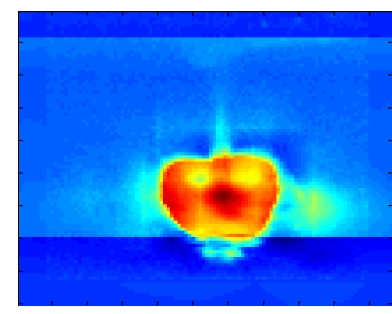

b)

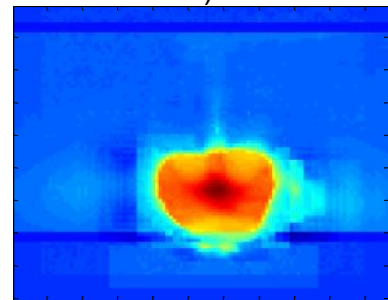

f)

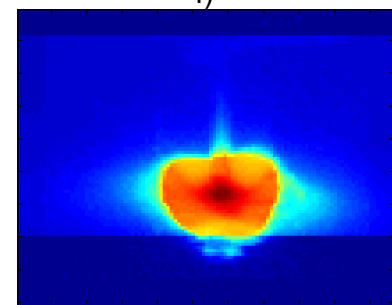

j)

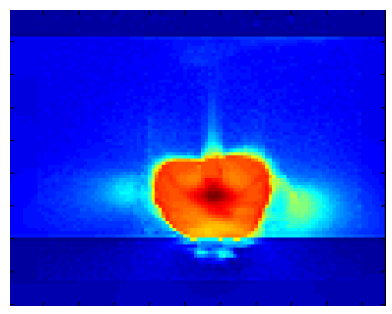

c)

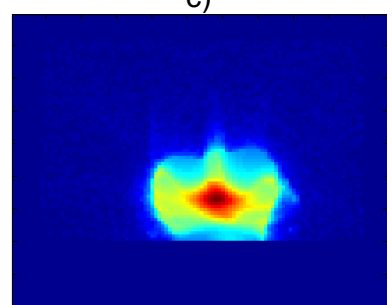

g)

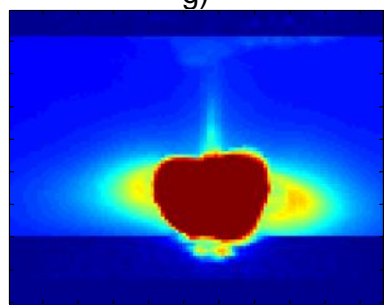

k)

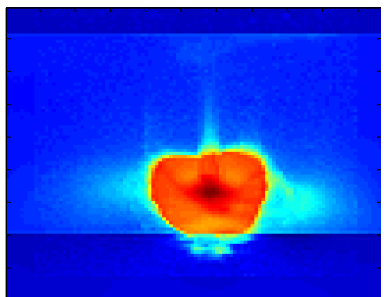

d)

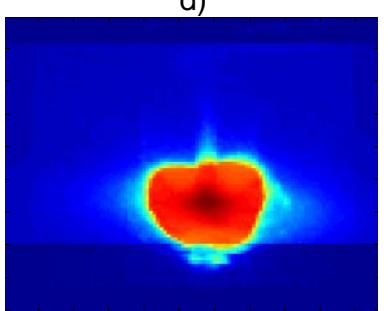

h)

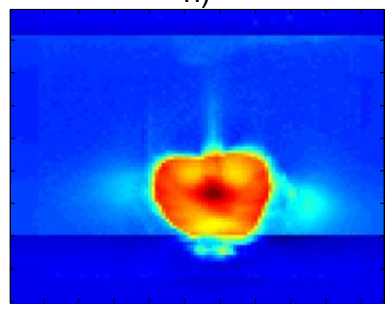

l)

Fig. 3. Examples of IR and TV images fused with methods: a) CON, b) DWB, c) FSD, d) GRA, e) $L A P$, f) MOD, g) MIN, h) $P C A$, i) $R A T$, j) MEA, k) MAX, I) $S I H$

\section{Evaluation of agregation algorithms}

The variety of image registration and aggregation methods makes the choice of the best fusion of visual and infrared images that is suitable for practical application in the process of arc welding state evaluation a difficult task. As it is shown in Fig. 3., each of the aggregation methods highlighted varying degree features specific to the visible light and infrared images. This indicates that taking of an optimal algorithm for aggregation must be carried out very carefully and be based on a multi-criteria evaluation. So far there is no universal method for efficient multi-criteria evaluation of aggregation methods for their use in generating synthetic images which could be useful in the automatic analysis of images taken during dynamic processes. The main problem in the development of good methods for assessing fusion algorithms is the difficulty in the choice of realistic assessment criteria, which usually requires the assumption of the reference image.

Depending on needs, assessment of the quality of synthetic images created during the fusion can be carried out in two ways: subjective and objective. In the subjective approach the images being the result of the fusion are assessed visually by the experts. They indicate images and their main features that are most relevant from the information point of view. So far this is the most effective way of assessing by assumption that the assessment is made for a small collection of images and expert has indisputable qualifications in the area governed by the content presented on the images. The second group of the methods provide an objective estimates. They consist in determining the values of numerical measures that are representative for the information content of images after the fusion. The difficulties in the practical application of the methods from this group could be the lack of direct relationship between the estimate values and relevant feature values which determine the suitability of the image for further analysis. The advantage of use of the 
objective methods giving numerical estimates is possibility to implement it in the computer program, which allows the automatic valuation of large collections of images resulted from the fusion process. The most popular objective measures are:

- Objective image fusion performance measure,

- Image quality index,

- Structural similarity,

- Mutual information,

- Mean squared error,

- Standard deviation,

- Entropy.

In the presented research only those methods were taken into consideration which do not need reference image. The chosen metrics are:

a. Standard deviation $(S)$ - is a very effective metric in the case of evaluation of images without blur and it allows the image contrast estimation. Image with higher contrast will be characterized by higher standard deviation value. Standard deviation is defined as follows

$$
\sigma=\sqrt{\sum_{i=0}^{L}(i-\bar{\imath})^{2} h_{I_{f}}(i)}
$$

where: $h_{I_{f}}(i)$ is the histogram of aggregated image $I_{f}$, and $L$ is the number of histogram bins, equal to the number of image greyscale levels.

a. Entropy (En) -is used to determine the amount of information contained in the signal. If entropy of newcreated fused image is higher than the entropy of each input image, it can be stated that the fused image contains more information than given input images. Higher entropy value indicates best fusion quality. Entropy is defined in the following way:

$$
H=-\sum_{i=0}^{L} h_{I_{f}}(i) \log _{2}\left\{h_{I_{f}}(i)\right\}
$$

b. Fusion mutual information (Mi) - it measures the degree of dependence of two images. A larger measure implies better quality. It is calculated as a sum of mutual information between input images $I_{1}$ and $I_{2}$ and fused image $I_{f} \cdot M i_{F}$ is given by:

$$
M i_{F}=M i_{I_{1} I_{f}}+M i_{I_{2} I_{f}}
$$

where mutual information between two images is equal to:

and $h_{I_{1} I_{2}}(i, j)$ is the joint histogram between $I_{1}$ and $I_{2}$.

$$
M i_{I_{1} I_{2}}=\sum_{i=0}^{L} \sum_{j=0}^{N} h_{I_{1} I_{2}}(i, j) \log _{2}\left\{\frac{h_{I_{1} I_{2}}(i, j)}{h_{I_{1}}(i, j) h_{I_{1}}(i, j)}\right\}
$$

c. Objective image fusion performance measure (Q) [31] - is a metric, which measures the amount of potentially important edge information 'transferred' from the source image to the fused image. The measure takes values in the range from 0 to 1 . Value 0 , means that no edge information from input images is preserved in the output image. For source images $A$ and $B$ and the output image being the result of $A$ and $B$ fusion $F$ is calculated in the following way:

- $\quad$ Computation of edge strength map $g(n, m)$ and edge orientation map $\alpha(n, m)$ for each pixel $p(n, m)$, for each input image where $n, m$ are indices of image pixels. For the image $A$ edge strength and orientation maps are defined as:

$$
\begin{aligned}
& g_{A}(n, m)=\sqrt{s_{A}^{x}(n, m)^{2}+s_{A}^{y}(n, m)^{2}} \\
& \alpha_{A}(n, m)=\tan ^{-1}\left(\frac{s_{A}^{y}(n, m)}{s_{A}^{x}(n, m)}\right)
\end{aligned}
$$

where: $s_{A}^{x}(n, m)$ and $s_{A}{ }^{y}(n, m)$ is the result of horizontal and vertical Sobel operator for pixel $p(n, m)$ in image $A$

- $\quad$ Relative strength $G^{A F}(n, m) i G^{B F}(n, m)$ and orientation $A^{A F}(n, m) A^{B F}(n, m)$ maps between input images $A$ i $B$, and the fused images $F$. Below the way in which the above mentioned coefficients are calculated is shown for images $A$ and $F$. For $B$ and $F$ the calculation procedure is analogous. 


$$
\begin{gathered}
G^{A F}(n, m)= \begin{cases}\frac{g_{F}(n, m)}{g_{A}(n, m)} & , g d y g_{A}(n, m)>g_{F}(n, m) \\
\frac{g_{A}(n, m)}{g_{F}(n, m)} & , g d y g_{A}(n, m)<g_{F}(n, m)\end{cases} \\
A^{A F}(n, m)=\frac{|| \alpha_{A}(n, m)-\alpha_{F}(n, m)\left|-\frac{\pi}{2}\right|}{\frac{\pi}{2}}
\end{gathered}
$$

- $\quad$ These measures are then used to estimate the edge strength and orientation preservation values $Q_{g}{ }^{A F}(n, m)$ and $Q_{\alpha}{ }^{A F}(n, m)$ specifying amount of edge information from $A$, preserved in the fused output image $F$

$$
\begin{aligned}
& Q_{g}^{A F}(n, m)=\frac{\Gamma_{g}}{1+e^{\kappa_{g}\left(G^{A F}(n, m)-\sigma_{g}\right)}} \\
& Q_{\alpha}^{A F}(n, m)=\frac{\Gamma_{\alpha}}{1+e^{\kappa_{\alpha}\left(G^{A F}(n, m)-\sigma_{\alpha}\right)}} \\
& \Gamma_{g}=0,9994 ; \kappa_{g}=-15 ; \sigma_{g}=0,5 \\
& \Gamma_{\alpha}=0,9879 ; \kappa_{\alpha}=-22 ; \sigma_{\alpha}=0,8
\end{aligned}
$$

- $\quad$ The overall edge information preservation value is then defined as:

$$
Q^{A F}(n, m)=Q_{g}^{A F}(n, m) Q_{\alpha}^{A F}(n, m)
$$

- A normalised weighted quality metric is given as:

$$
Q^{A B / F}=\frac{\sum_{n=1}^{N} \sum_{m=1}^{M} Q^{A F}(n, m) w^{A}(n, m)+Q^{B F}(n, m) w^{B}(n, m)}{\sum_{i=1}^{N} \sum_{j=1}^{M}\left(w^{A}(i, j)+w^{B}(i, j)\right)}
$$

where: $W^{A}$ i $W^{B}$ are weighted by coefficients reflecting the perceptual importance of the corresponding edge elements within all input images.

\subsection{Selection of the best aggregation methods with the use of objective numerical descriptors}

To evaluate the images obtained as a result of application of 12 aggregation algorithms, four previously described measures of quality Me, Q, S, En were used. Estimated images represented 12 classes of state of the welding process (see section 2.1.). Evaluation of the fused images was performed for each class of state.

For each class statistics such as: mean, minimum, maximum and standard deviation were calculated. For better readability of results the obtained values were averaged regardless the state class.

Mean results obtained for the quality measures of each state class allowed the selection of a subset of the aggregation algorithms which were the most useful for fusion of the visual and infrared images of the welding arc. A subset of the best algorithm was selected on the basis of the first 4 highest values of considered quality measures. Table 1 presents the ordered results of the selection of aggregation algorithms, based on average values of quality measure.

Table 2. Highest rated aggregation methods

\begin{tabular}{|c|l|l|l|l|}
\hline \multirow{2}{*}{ Quality measure } & \multicolumn{4}{|c|}{ Usability rate } \\
\cline { 2 - 5 } & A & B & C & D \\
\hline Q & MAX & LAP & MOR & SIH \\
\hline S & MOR & RAT & MAX & LAP \\
\hline En & SIH & DWB & LAP & GRA \\
\hline Mi & MAX & PCA & MEA & MOR \\
\hline
\end{tabular}


It can be seen that each of the measures of quality has taken the highest values for the different aggregation algorithm. The best algorithm, according to measure Q and Mi was the point based MAX algorithm. Algorithms MOR and $\mathrm{SIH}$ can be regarded as the best on the basis of the highest measurement values of $S$ and En measures. A detailed analysis of a subset of selected aggregation algorithms presented in Table 1 allows the choice of three aggregation algorithms LAP, SIH, and MOR, indicated as the best by most of the considered measures of quality. It can be also assumed that the aggregation algorithms such as wavelet DWD, pyramid RAT, and the PCA and spot MEA may prove useful in the process of fusion of visual and infrared welding arc images. The values of quality measures were determined for different images representing different classes of state. This allowed examination of the scatter of measurements for each method of aggregation. Rating of measurement scatter values allows us to specify aggregation algorithms that are independent from changes in the input images. Table 2 presents algorithms for which the dispersion of the values of quality metrics was smallest, Figure 2 shows how the minimum and maximum values evolved for the various measures of the quality of aggregation algorithms.

Table 3. Methods with smallest image quality metrics scatter

\begin{tabular}{|c|l|l|l|l|}
\hline \multirow{2}{*}{ Quality measure } & \multicolumn{4}{|c|}{ Usability rate } \\
\cline { 2 - 5 } & A & B & C & \multicolumn{1}{|c|}{ D } \\
\hline Q & MEA & SIH & CON & GRA \\
\hline S & FSD & GRA & MEA & DWB \\
\hline En & MIN & CON & MAX & LAP \\
\hline Mi & SIH & DWB & FSD & MOR \\
\hline
\end{tabular}
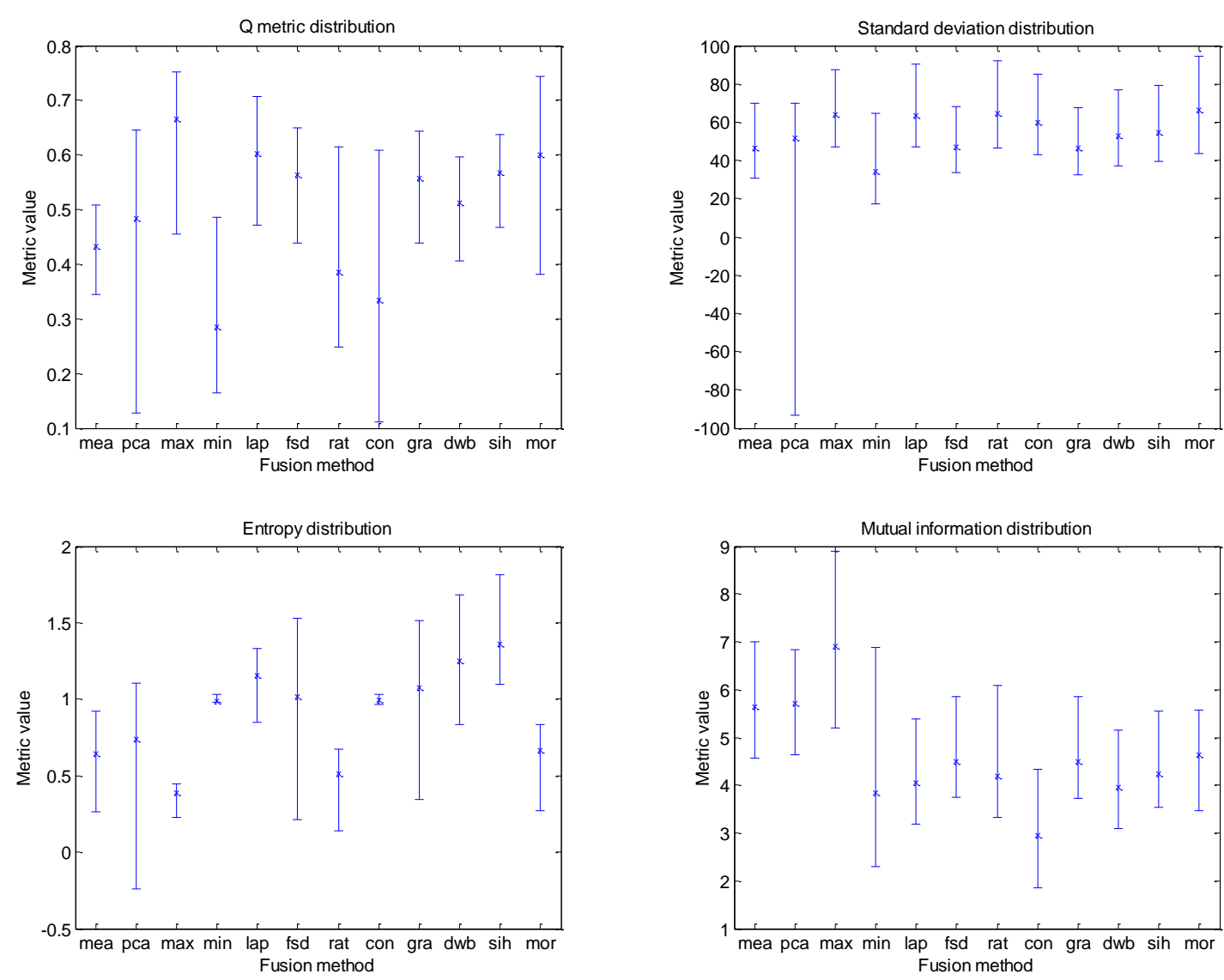

Fig. 4. Scatter of the image quality measure values 

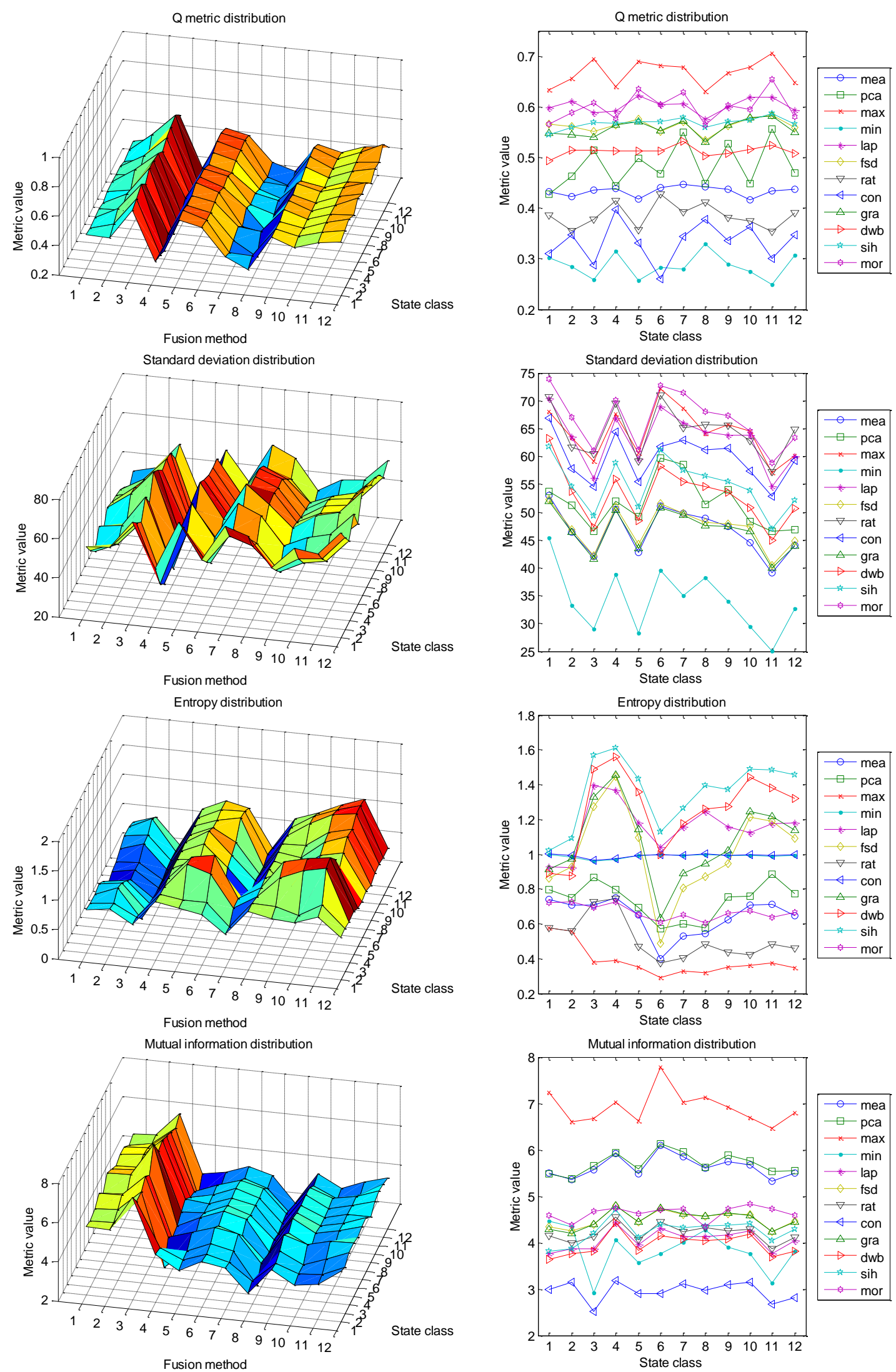

Fig. 5. Values of quality measures in relation to considered fusion methods and state classes 
It is easy to notice that the values of quality measures for aggregation algorithms LAP, MOR and MAX previously recognized as the best, have a relatively large spread of values. The smallest scatter of the measure values was observed for the aggregation algorithms, which gives the lowest mean values, being least useful fusion of infrared and visible light images. It can be said that the fluctuation in the measure value does not retain the symmetry relative to the mean. There is a clear tendency to obtain higher values of measurement, that is bounded to the fact that often generated are better images than the worse from of an objective assessment point of view. On the basis of the results shown in Tab. 2 it can be found that the best algorithms in terms of the smallest scatter of the quality measure values can be regarded the wavelet algorithms DWB, SIH, and gradient pyramid algorithm GRA.

During the evaluation of algorithms, the variability of each measurement according to class of the welding process state was also analysed. In Fig. 3 presents the three-dimensional graphs showing changes of values of the quality measures as a function of the welding process state class and type of aggregation algorithm. 2D drawings show more accurately the variability of measure obtained for each algorithm depending on the state class. In addition, Table 3 lists the standard deviations for each measure and each of the considered aggregation algorithms.

Table 4. Standard deviations for considered measure for aggregation algorithms

\begin{tabular}{|l|l|l|l|l|}
\hline AlgorithmIMetric & Q & S & En & Mi \\
\hline MEA & 0,023 & 7,248 & 0,141 & 0,377 \\
\hline PCA & 0,108 & 9,120 & 0,279 & 0,293 \\
\hline MAX & 0,060 & 6,031 & 0,045 & 0,489 \\
\hline MIN & 0,062 & 9,906 & 0,009 & 0,900 \\
\hline LAP & 0,041 & 7,889 & 0,074 & 0,399 \\
\hline FSD & 0,039 & 6,336 & 0,221 & 0,375 \\
\hline RAT & 0,067 & 7,661 & 0,067 & 0,484 \\
\hline CON & 0,088 & 7,561 & 0,009 & 0,428 \\
\hline GRA & 0,037 & 6,289 & 0,192 & 0,374 \\
\hline DWB & 0,030 & 7,284 & 0,129 & 0,366 \\
\hline SIH & 0,028 & 7,229 & 0,107 & 0,349 \\
\hline MOR & 0,071 & 8,780 & 0,082 & 0,314 \\
\hline
\end{tabular}

Plots of measures of quality and value of their standard deviations indicate that the most stable, depending on the considered state class are measurement of $Q$ and $M$. Thus, these measurements can be assumed to be the most representative for the assessment of aggregation algorithms of infrared and visible light images of the welding arc.

Taking into account the results of the values of objective measures, it was found that the most useful of the aggregation algorithm of infrared and visible light images of the welding arc is hierarchical morphological differences algorithm MOR.

\subsection{Subjective images assessment}

Subjective evaluation of aggregation algorithms was performed by three independent experts in the field of welding technology. The experts evaluated four sets of fused images. Each set consists of 12 images created by means of considered aggregation method of vision and infrared images acquired in different states of the welding process. Each expert indicated four best in his opinion images and rated them using scale from A to $D$. The main criterion which the experts used was visibility of image features, which allowed them to identify phenomena occurring in welding arc area. The authors did not perform induction of decision rules which the experts used.

The analysis of the gathered expert rates allowed us to indicate the best 4 fusion algorithms in the sense of subjective evaluation. The identifiers of selected algorithms were presented in Table 5. It can be clearly seen that the algorithm MEA was most often indicated by experts. Similarly, images resulting from the use of algorithms SIH, RAT, GRA and MAX were also frequently selected.

Table 5. Results of subjective evaluation of image fusion algorithms

\begin{tabular}{|c|l|c|c|c|}
\hline \multirow{2}{*}{ Expert Id } & \multicolumn{4}{|c|}{ Usability rate } \\
\cline { 2 - 5 } & A & B & C & D \\
\hline E1 & MEA & SIH & RAT & GRA \\
\hline E2 & SIH & MEA & MAX & RAT \\
\hline E3 & DWB & GRA & MAX & MEA \\
\hline Global & MEA & SIH & RAT & GRA \\
\hline
\end{tabular}




\section{Conclusions}

The article shows results of the research which aims at indicating the subset of at least two from 12 best aggregation algorithms in order to apply them in fusion process of vision and infrared images of welding arc. Selection was performed on the basis of objective and subjective evaluation of images of welding arc resulted from the use of fusion of vision and infrared images acquired in 12 different states of welding process.

The objective evaluation was made by means of 4 quantitative quality measures. The analysis of estimate values allowed indicating three best algorithms which were MAX, MOR and SIH. Additionally, scatter of estimate values was analysed which showed the best three algorithms with the smallest scatter of estimates values, which were DWB, SIH and GRA. Evaluation of scatter of measure values according to considered classes of welding process state gives us a conclusion that measures $\mathrm{Q}$ and $\mathrm{Mi}$ are the most adequate for estimation of welding arc images resulting from fusion operation.

Objective evaluation performed by three experts of welding technology indicated five best fusion algorithms which are MEA, SIH, RAT, GRA and MAX. The results are convergent to the results of objective estimation with the use of measures $\mathrm{Q}$ and $\mathrm{Mi}$.

Taking into consideration all results of evaluation of fusion algorithms it can be stated that SIH and GRA are the most suitable aggregation algorithms for use in fusion process of vision and infrared images of welding arc. In the authors' opinion these algorithms subset should be completed with algorithms based on wavelet transformation like MOR and DWB.

The performed research showed that despite the fact that subjective and objective estimates are convergent, it is necessary to elaborate an additional quality measure which would allow quality estimation of images of peculiar features describing a process i.e. welding process features. Searching for quality measure suitable for estimation of images of welding arc after fusion will be the aim of the further research of the authors.

\section{REFERENCES}

[1] Al-Karawi, J., Schmidt, J., "Application of Infrared Thermography to the analysis of Welding processes". 7th International Conference on Quantitative Infrared Thermography, Belgium, July 5-8, 2004, H.9.1-H.9.6.

[2] Anderson $\mathrm{H}$., "A filter-subtract-decimate hierarchical pyramid signal analyzing and synthesizing technique". U.S. Patent 718 104, 1987.

[3] Balfour C., Smith J.S., "Amin-Nejad S.: Feature correlation for weld image-processing applications", International Journal of Production Research, vol. 42, no. 5, pp. 975-995, 2004.

[4] Bracewell R.N., "The Fourier Transform and Its Applications". McGraw-Hill, New York, 1965.

[5] Burt P., Adelson E., "Laplacian pyramid as a compact image code". IEEE Transactions on Communications, vol. 31, no. 4, 1983.

[6] Burt P., Kolczynski P., "Enhanced image capture through fusion," in Proc. 4th Int. Conf. Computer Vision, pp. 173-182, 1993.

[7] Camilleri D., Gray T., and Comlekci T., "Use of Thermography to Calibrate Fusion Welding Procedures in Virtual Fabrication Applications". Proc. of Inframation 2004 Conference, vol. 5 pp. 121 -131, 2004.

[8] Chen H.-M. and Varshney, P.K., "Automatic two-stage IR and MMW image registration algorithm for concealed weapons detection". Vision, Image and Signal Processing, IEEE Proceedings, vol.148, no.4, pp.209-216, 2001.

[9] Cook, G.E., Barnett, R.J., Andersen, K., Springfield, J.F., Strauss, A.M., "Automated visual inspection and interpretation system for weld quality evaluation". Thirtieth IAS Annual Meeting, IAS '95., vol.2, pp.1809-1816, 1995.

[10] Govardhan S.M., Wikle H.C., Nagarajan C., and Chin B.A., "Real-Time Welding Process Control Using Infrared Sensing". Proceedings of the American Control Conference, pp. 1712-1716, 1995.

[11] Huang R., Liu L., and Song G., "Infrared temperature measurement and interference analysis of magnesium alloys in hybrid laser-TIG welding process". Materials Science and Engineering: A, vol. 447, pp. 239-243, 2007.

[12] Irani M. and Anandan P., "Robust multi-sensor image alignment". in Proc. Int. Conf. Computer Vision, pp. 959966, 1998.

[13] iShot@ Weld-i Automated Weld Viewer System http://www.remotevisionsource.com/, 2011.03.07

[14] Jamrozik W., Fidali M., Registration methods for infrared and vision images representing dynamically changing scene, Pomiary Automatyka Kontrola, vol. 57, no. 10, pp. 1134 - 1137, 2011 (in polish).

[15] Kim J.S., Son Y.T., Cho H.S., Koh K.II.: A robust method for vision-based seam tracking in robotic arc welding, Proceedings of the IEEE International Symposium on Intelligent Control, 1995

[16] Kim Y. S. and Lee J. H. and Ra J. B., 2008, Multi-sensor image registration based on intensity and edge orientation information, Pattern Recognition, vol. 41, pp. 3356-3365

[17] Li, S. T., Wang, Y. N., 2000, Multisensor image fusion using discrete multiwavelet transform, Proceedings of the 3rd International Conference on Visual Computing," Mexico City, Mexico.

[18] Lucas W., Smith J., Balfor C., Bertaso D., Melton G., „Real-time vision based control of weld pool size”. Przegląd Spawalnictwa vol. 81, no. 1, pp. 11-16, 2009 (in polish). 
[19] Malamas E.N., Petrakis E.G.M., Michalis Zervakis, Petit L., Legat J.D., "A survey on Industrial Vision systems application tools". Image and vision computing, vol. 21, pp. 171-188, 2003

[20] Metwalli M. R., Nasr A.H., Farag Allah O.S., El-Rabaie S., "Image fusion based on principal component analysis and high-pass filter". International Conference on Computer Engineering \& Systems, 2009. ICCES 2009, pp.6370, 2009.

[21] Noruk J., Boillot J.P., "Laser Vision Technology Ensures Six Sigma-level Quality is Achieved in Robotic Welding". Canadian Welding Association Journal, vol. 8, pp. 8-14, 2006.

[22] Ramac, L. C., Uner, M. K., Varshney, P. K., "Morphological filters and wavelet based image fusion for concealed weapon detection". Proceedings of SPIE, vol. 3376, pp. 110-119, 1998.

[23] Rockinger, O., "Image Sequence Fusion Using a Shift Invariant Wavelet Transform". Proceedings of the International Conference on Image Processing, vol.3, pp. 288-291, 1997.

[24] Saeedi, J.; Faez, K., "The new segmentation and fuzzy logic based multi-sensor image fusion". 24th International Conference Image and Vision Computing New Zealand, 2009. IVCNZ '09., pp. 328-333, 2009.

[25] Smith J.S., Balfour C., "Real time top-face vision based control of weld pool size". Industrial Robot: An International Journal, vol. 32, pp. 334 - 340, 2005.

[26] Stathaki T., Image Fusion: Algorithms and Applications, Academic Press, 2008

[27] Thamilarasi, P.; Ragunathan, S.; Mohankumar, E., "Automation of robot welding using Machine vision - A review"., International Conference on Communication and Computational Intelligence (INCOCCI), pp. 266-268, 2010

[28] Toet A., "Image fusion by a ratio of low-pass pyramid". Pattern Recognition Letters 9, pp. 245-253, 1996.

[29] Toet A., van Ruyven J. J. and J. M. Valeton, "Merging thermal and visual images by a contrast pyramid". Optical Engineering, vol. 28, no. 7, pp. 789-792, 1989.

[30] Węglowski M., "Utilization of the arc light emission emitted during TIG welding to monitoring this process". Archives of Mechanical Technology and Automation PAN, vol. 27, no. 1, pp. 103-112, $2007 . \quad$ (in Polish).

[31] Xydeas, C.S.; Petrovic, V., "Objective image fusion performance measure". Electronics Letters, vol.36, no.4 pp.308-309, 2000.

[32] B. Zitová, J. Flusser, "Image registration methods: a survey". Image Vision Comput. vol. 21 no.11, pp. 9771000, 2003. 